tolerance and the development of systemic autoimmune disease.

Objectives To determine the extent of cellular senescence of LNSCs during early phases of systemic autoimmunity.

Methods We included individuals with arthralgia without any evidence of arthritis who were positive for IgM rheumatoid factor (IgM-RF) and/or anti-citrullinated protein antibodies (ACPA; RA-risk group), early arthritis patients (ACR/EULAR 2010 criteria; disease duration $<1$ year) and seronegative healthy controls. All study subjects underwent ultrasoundguided inguinal lymph node biopsy. LNSCs were cultured from freshly collected lymph node needle biopsies and passages $0-9$ were used for experiments. Flow cytometry, qPCR and microscopy were used to measure cell size, granularity, senescence-associated gene expression levels, telomere attrition and senescence-associated $\beta$-galactosidase (SA $\beta$-gal) activity.

Results Preliminary flow cytometry data shows that the cell size of LNSCs from RA patients $(\mathrm{n}=11)$ and RA-risk individuals $(n=7)$ is increased compared with healthy LNSCs $(n=7)$, while granularity was specifically increased in LNSCs from RA patients $(n=9)$. Initial SA $\beta$-gal stainings indicate higher activity in LNSCs from RA-risk $(n=3)$ and RA patients $(n=4)$ compared with healthy controls $(n=2)$, however this data needs to be carefully interpreted and more donors should be analysed. Expression levels of senescence-associated genes significantly increased over culture passages and significantly higher p21 and p53 levels were observed in passage 9 LNSCs from RA patients compared with healthy controls ( $n=6$ per group).

Conclusions These preliminary findings provide a rationale for studying cellular senescence in LNSCs in more detail during different phases of RA and to investigate the consequence of senescent LNSCs on immune cell responses upon their interaction.

Disclosure of Interest None declared.

\section{P113/017 HUMAN LYMPH NODE STROMAL CELLS EXPRESS SELF- ANTIGENS TARGETED BY ANTI-CITRULLINATED PROTEIN ANTYBODIES: ROLE FOR TOLERANCE INDUCTION IN RHEUMATHOID ARTHRITIS}

\begin{abstract}
${ }^{1,2} \mathrm{C}$ Grasso*, 1,2 JS Hähnlein, ${ }^{3} \mathrm{R}$ Nadafi, 1,2TA De Jong, 1,2 $\mathrm{TH}$ Ramwadhdoebe, 1,2JF Semmelink, ${ }^{2} \mathrm{DM}$ Gerlag, ${ }^{2} \mathrm{PP}$ Tak, ${ }^{3} \mathrm{RE}$ Mebius, ${ }^{2} \mathrm{M}$ Safy, ${ }^{4} \mathrm{M}$ Maas, ${ }^{5} \mathrm{Al}$ Catrina, ${ }^{5} \mathrm{H}$ Wähämaa, ${ }^{1,2} \mathrm{LGM}$ Van Baarsen. ${ }^{1}$ Amsterdam Rheumatology and immunology Center (ARC), Academic Medical Center; ${ }^{2}$ Department of Rheumatology and Clinical Immunology, the Department of Experimental Immunology, Amsterdam Infection and Immunity Institute, Amsterdam UMC, University of Amsterdam; ${ }^{3}$ Department of Molecular Cell Biology and Immunology, Amsterdam UMC, Vrije Universiteit Amsterdam,VU medical center; ${ }^{4}$ Depertment of Radiology, Amsterdam UMC, University of Amsterdam, Amsterdam, Netherlands; ${ }^{5}$ Rheumatology Unit, Department of Medicine, Sweden, Karolinska University Hospital and Karolinska Institutet, Stockholm, Sweden
\end{abstract}

\subsection{6/annrheumdis-2018-EWRR2019.101}

Career situation of first and presenting author Student for a master or a PhD.

Introduction In rheumatoid arthritis (RA) the cause for loss of tolerance and anti-citrullinated protein antibody (ACPA) production remains unidentified. Mouse studies showed that peripheral tolerance can be maintained through presentation of peripheral tissue antigens (PTAs) by lymph node stromal cells (LNSCs). We hypothesize that deregulation of peripheral tolerance mechanisms mediated by LNSCs might underlie pathogenesis of RA. Here we investigated the expression of PTAs, citrullinated proteins and immunomodulatory molecules by human LNSCs during health and autoimmunity.

Methods LN tissue sections and LNSCs were prepared from freshly collected lymph node needle biopsies obtained from 24 patients with RA, 23 individuals positive for autoantibodies but without clinical apparent disease (RA-risk group) and 14 seronegative healthy controls. Expression of PADI enzymes, citrullinated proteins, DEAF1, AIRE and PTAs was investigated at mRNA and protein level. Expression of immunomodulatory molecules in LNSCs was assessed after stimulation with IFN $\gamma$ $(\mathrm{n}=15)$.

Results Citrinullated proteins, targeted by ACPA, were found in human LN tissue as well as in cultured LNSCs. In addition, we observed the expression of transcription factors AIRE and DEAF1 as well as disease-related PTAs in LNSCs with some PTAs showing a distinct expression pattern in autoimmune LNSCs compared to healthy controls. TGF- $\beta$ was constitutively expressed by LNSCs while CD86 or IL-10 were not detected. Upon IFN $\gamma$ stimulation LNSCs upregulated MHC class II, co-stimulatory molecules CD40 and CD80 as well as $\mathrm{T}$ cell negative regulators CD274, NOS2 and IDO. Overall, no clear differences between donor groups were observed for these markers with exception of a slightly lower induction of CD40 and NOS2 in RA LNSCs.

Conclusions We present for the first time that human LNSCs express several PTAs and the transcription factors AIRE and DEAF1, driving PTA expression. Additionally, human LNSCs express molecules involved in citrullination, antigen presentation and immunomodulation. Moreover, antigens targeted by ACPAs are present in LN tissue and LNSCs. These data suggest that LNSCs have the machinery to regulate peripheral tolerance.

Disclosure of Interest None declared.

\section{P114 TISSUE REGENERATION AND BONE REPAIR UPON TNF BLOCKADE IN EXPERIMENTAL ARTHRITIS}

K Wanic, on behalf of First author, M Kalkgruber, B Niederreiter, T Shvets, J Smolen, S Hayer*. Medical University of Vienna, Dep Medicine III, Div Rheumatology, Vienna, Austria

\subsection{6/annrheumdis-2018-EWRR2019.102}

Career situation of first and presenting author Student for a master or a $\mathrm{PhD}$.

Introduction Partial bone repair upon treatment with biological DMARDs has been reported in patients with Rheumatoid Arthritis (RA). Previous in vivo PET/CT studies using the human tumor necrosis factor transgenic mouse model (hTNFtg) have been demonstrated the reversibility of preexisting inflammatory, erosive arthritis with complete remission of synovial inflammation and repair of bone erosions upon TNF blockade.

Objectives To investigate spontaneous repair and regenerative processes of structural joint damage in ankle and knee joint, menisci and patella tendon upon TNF blockade in hTNFtg mice.

Methods Two cohorts of arthritic hTNFtg mice were treated with anti-TNF ab (infliximab, $10 \mathrm{mg} / \mathrm{kg}, 3 \mathrm{x}$ per week, i.p., 
4 weeks): (I) 8 week old mice with established inflammatory, erosive arthritis and (II) 12 week old hTNFtg mice with severe inflammatory, erosive arthritis $(\mathrm{n}=8-10$ animals). Clinical signs of arthritis were weekly assessed. Knee and ankle joints were used for $\mu \mathrm{CT}$ (Scanco $\mu \mathrm{CT} 35$ ) and subsequent histology. Toluidine blue staining indicated proteoglycan contents, tartrate-resistant acid phosphatase staining identified osteoclasts. Immunohistochemical stainings were performed for collagen type II (col II) and osterix. Expression of chondrogenic markers was analysed from RNA paw extracts.

Results TNF blockade significantly improved clinical signs in both treatment cohorts. Compared to eroded bone surfaces at week 8 (before treatment), TNF blockade led to improved bone architecture with regular, smooth bone surfaces in CT scans from knee and ankle. Histologically, treatment allowed complete resolution of synovitis, osteoclast activity and subchondral bone erosions. Despite sites of intact bone tissue, regenerative processes of cortical and subchondral bone were found. Erosions of bone and calcified cartilage were filled with col II positive cartilaginous or fibro-cartilaginous tissue. Hyperproliferative chondrocytes and endochondral ossifications were found in knees. Consistently, chondrogenic markers like Sox9 transcription factor and col II were markedly increased, accompanied by decreased expression of cartilage degrading enzymes (MMP3, MMP13) upon treatment. Of note, menisci and patella tendon showed massive hyperproliferative chondrocytes, col II and osterix staining.

Anti-TNF treatment at later stages with severe bone destruction (at week 12) could not completely resolve synovitis and restore structural damage. Pannus tissue transformed into chondrocyte rich, fibrocartilaginous tissue. Callus and ossifications were formed in knees.

Conclusions Therapeutic TNF blockade of inflammationmediated joint damage enables the initiation of directed but also undirected regenerative processes. Remission of synovitis seems to be essential to promote bone and calcified cartilage repair.

Disclosure of Interest None declared.

\section{P115/019 IMMUNOMODULATORY ACTIVITY OF ADIPOSE- DERIVED MESENCHYMAL STEM CELLS OF ANKYLOSING SPONDYLITIS PATIENTS}

${ }^{1}$ E Kuca-Warnawin*, ${ }^{1} \mathrm{U}$ Skalska, ${ }^{1} \mathrm{I}$ Janicka, ${ }^{2} \mathrm{~K}$ Bonek, ${ }^{2} \mathrm{P}$ Głuszko, ${ }^{1} \mathrm{~W}$ Maslinski, ${ }^{1} \mathrm{E}$ Kontny. ${ }^{1}$ Department of Pathophysiology and Immunology; ${ }^{2}$ Department of Rheumatology, National Institute of Geriatrics, Rheumatology, and Rehabilitation, Warsaw, Poland

\subsection{6/annrheumdis-2018-EWRR2019.103}

Career situation of first and presenting author Post-doctoral fellow.

Introduction Ankylosing spondylitis (AS) is a chronic autoimmune disease characterized by inflammation and pathological new bone formation at axial joints with resulting spinal segments fusion. The immune abnormalities observed in AS patients concern mainly $\mathrm{T}$ lymphocytes and manifest by increased numbers of circulating Th17 cells and defects of regulatory $\mathrm{T}$ (Treg) cells. Adipose mesenchymal stromal/stem cells (ASCs) exert immunosuppressive effects on different immune cells, including $\mathrm{T}$ lymphocytes, and may be promising option for successful therapy of AS.

Objectives In this study we have focused on immunoregulatory activity of ASCs from AS patients (AS/ASCs) toward healthy donor peripheral blood mononuclear cells (PBMCs) and $\mathrm{T}$ lymphocytes with special regard to Th17 and Treg cell subsets.

Methods AS/ASCs of 15 AS patients and commercially available ASC lines from healthy donors (hASC) were used. ASCs were co-cultured with either anti-CD3/CD28stimulated $\mathrm{CD}^{+} \mathrm{T}$ lymphocytes or mitogen-stimulated PBMCs of allogeneic healthy volunteers. Expression of CD25 and FoxP3 transcription factor in $\mathrm{T}$ cells and these cells proliferation were evaluated by flow cytometry. Concentrations of cytokines associated with Th1 (IFN $\gamma$ ), Th17 (IL-17AF) or Treg (IL-10, TGFß) cells as well as of factors related to immunomodulatory function of ASCs (PGE 2 , kynurenines) were measured by ELISAs in co-culture supernatants.

Results In the co-cultures of ASCs with PBMCs both hASCs and AS/ASCs exerted similar inhibitory effect on the proliferation response of $\mathrm{T} \mathrm{CD}^{+}$and $\mathrm{CD}^{+}$cells. Similar results were obtained in co-cultures of ASCs with purified $\mathrm{T} \mathrm{CD} 4^{+}$cells. In the co-cultures of hASCs and AS/ASCs with PBMCs as well as with purified $\mathrm{T} \mathrm{CD}^{+}$cells the significant increases of kynurenines and $\mathrm{PGE}_{2}$ production were observed. In co-cultures of ASCs and PBMCs there was a marked decrease of IFNg and significant increase of IL-17AF. Concomitantly, Treg proportion in these co-cultures was elevated. In contrary, in the co-cultures of activated $\mathrm{T} \mathrm{CD}^{+}$cells with ASCs there was some increase of IFNg and IL-17AF whereas Treg proportion was downregulated.

Conclusions AS/ASCs and hASCs exert comparable effects on $T$ cells. Since different effects were observed in the co-cultures of ASCs with PBMCs than with $\mathrm{CD}^{+}$cells, it seems that ASCs activity toward Th17 and Treg cells is dependent on the presence of accessory cells.

Acknowledgements This work was supported by the National Science Centre in Poland (Grant No. 2016/21/B/ NZ5/00500) and by the NIGRiR Statutory Grant (Grant No. S/6).

Disclosure of Interest None declared.

\section{P116 BASIC CHARACTERISTICS OF ADIPOSE-DERIVED MESENCHYMAL STEM CELLS OF ANKYLOSING SPONDYLITIS PATIENTS}

${ }^{1}$ E Kuca-Warnawin*, ${ }^{1} \mathrm{U}$ Skalska, ${ }^{1} \mathrm{M}$ Plebanczyk, ${ }^{1} \mathrm{I}$ Janicka, ${ }^{1} \mathrm{U}$ Musialowizz, ${ }^{2} \mathrm{~K}$ Bonek, ${ }^{2} \mathrm{P}$ Głuszko, ${ }^{1} \mathrm{E}$ Kontny. ${ }^{1}$ Department of Pathophysiology and Immunology; ${ }^{2}$ Department of Rheumatology, National Institute of Geriatrics, Rheumatology, and Rehabilitation, Warsaw, Poland

\subsection{6/annrheumdis-2018-EWRR2019.104}

Career situation of first and presenting author Post-doctoral fellow. Introduction Application of mesenchymal stem/stromal cells (MSCs), endowed with immunosuppressive and regenerative properties, may be promising option for successful therapy of ankylosing spondylitis (AS) patients, characterised by inflammation and pathological bone remodelling. Adipose tissue is an 